OPEN ACCESS

Edited by:

Roberta Sellaro,

Leiden University, Netherlands

Reviewed by:

Luisa Lugli,

University of Bologna, Italy

Hayward James Godwin,

University of Southampton, UK

*Correspondence:

Carlos Velasco

Crossmodal Research Laboratory,

Department of Experimental

Psychology, University of Oxford,

9 South Parks Road,

Oxford OX1 3UD, UK

carlos.velasco@psy.ox.ac.uk

Specialty section:

This article was submitted to

Cognition, a section of the journal

Frontiers in Psychology

Received: 05 January 2015 Accepted: 02 March 2015

Published: 27 March 2015

Citation:

Velasco C, Wan X, Knoeferle K,

Zhou X, Salgado-Montejo A and Spence $C$ (2015) Searching for flavor labels in food products: the influence

of color-flavor congruence and

association strength.

Front. Psychol. 6:301.

doi: 10.3389/fpsyg.2015.00301

\section{Searching for flavor labels in food products: the influence of color-flavor congruence and association strength}

\author{
Carlos Velasco ${ }^{1 *}$, Xiaoang Wan², Klemens Knoeferle ${ }^{3}, \mathrm{Xi} \mathrm{Zhou}^{2}$, \\ Alejandro Salgado-Montejo ${ }^{1,4}$ and Charles Spence ${ }^{1}$
}

${ }^{1}$ Crossmodal Research Laboratory, Department of Experimental Psychology, University of Oxford, Oxford, UK, ${ }^{2}$ Tsinghua University, Beijing, China, ${ }^{3}$ Department of Marketing, BI Norwegian Business School, Oslo, Norway, ${ }^{4}$ Escuela Internacional de Ciencias Económicas y Administrativas, Universidad de La Sabana, Chía, Colombia

Prior research provides robust support for the existence of a number of associations between colors and flavors. In the present study, we examined whether congruent (vs. incongruent) combinations of product packaging colors and flavor labels would facilitate visual search for products labeled with specific flavors. The two experiments reported here document a Stroop-like effect between flavor words and packaging colors. The participants were able to search for packaging flavor labels more rapidly when the color of the packaging was congruent with the flavor label (e.g., red/tomato) than when it was incongruent (e.g., yellow/tomato). In addition, when the packaging color was incongruent, those flavor labels that were more strongly associated with a specific color yielded slower reaction times and more errors (Stroop interference) than those that were less strongly tied to a specific color. Importantly, search efficiency was affected both by color/flavor congruence and association strength. Taken together, these results therefore highlight the role of color congruence and color-word association strength when it comes to searching for specific flavor labels.

Keywords: flavor words, color, congruence, classification, visual search

\section{Introduction}

According to Garber et al. (2000), most food brands utilize color in one way or another in order to indicate the flavor of the products that they sell. Indeed, when it comes to food, visual cues, together with orthonasal olfaction, are thought to provide most of the information that is available to people before they eat and drink (see also Hutchings, 1977; Stevenson, 2009; Shankar et al., 2010). Color, in particular, may be one of the most important cues guiding people's flavor expectations, classification, search, and perception (see Stillman, 1993; Garber et al., 2000, 2001; Spence et al., 2010, 2014; Piqueras-Fiszman and Spence, 2011; Velasco et al., 2013).

Flavor information is also often conveyed by means of other design elements on food packaging (Garber et al., 2000). For example, the name of the flavor written on the package, or flavor label, can be important when it comes to deciding which product to buy, since color schemes can vary as a function of brand, product category, and country (e.g., Madden et al., 2000; Piqueras-Fiszman et al., 2012; Velasco et al., 2014). Notably, the congruency (e.g., consistency of the information in terms of consumers' prior experience) between the elements of marketing communications, such 
as a product's packaging flavor label and color, can be critical since congruent (e.g., tomato and red) and incongruent (e.g., tomato and blue) information may have different effects on consumer behavior (Mandler, 1982; Heckler and Childers, 1992; Peracchio and Tybout, 1996; Schoormans and Robben, 1997; Miller and Kahn, 2005).

People match both flavor names (e.g., Piqueras-Fiszman and Spence, 2011; Wan et al., 2014a) and actual flavors (e.g., Zampini et al., 2007; Wan et al., 2014b) to colors in specific ways. The way in which colors and flavors are associated are sensitive to a number of contextual variables (e.g., Shankar et al., 2010). This is presumably attributable to the internalization of the statistical regularities of the environment (Spence, 2011, 2012). Such an idea may explain why it is that certain associations, such as tomato with red, or cucumber with green (natural pairings), are consistent across countries, whereas others are not (the color associated to chicken-flavored crisps can vary across countries, Velasco et al., 2014). A key question here concerns whether color-flavor associations (and the strength of those associations) influence the classification and search for products with specific flavor labels, since the same product/flavor can often be presented in different colors, even in the same country (not to mention new products or colors that may be introduced to the market place).

The literature on the Stroop effect has already demonstrated that when a word (e.g., color name) does not match the (semantically related) color in which the word is presented, people take longer and make more errors when responding to the color of the word (Stroop interference, see MacLeod, 1991, for a review). Importantly, previous studies have demonstrated that the strength of the association between a color and a word can influence the magnitude of interference (Klein, 1964; Scheibe et al., 1967; Proctor, 1978; Klopfer, 1996; Berthet et al., 2011), which may extend to color-flavor word associations. Association strength between color and flavor words is crucial in the context of food products as some flavors may have a stronger color identity (e.g., strawberries) than others (e.g., chicken, see also Tanaka and Presnell, 1999; Lewis et al., 2013, on object color diagnosticity). The strength of the color identity may, in turn depend, for example, on the product category (e.g., an actual chicken has specific colors, which may be different when used in chicken-flavored crisp packaging).

Relevant to the context of the present study, a reversed Stroop effect, in which color interferes with the reading of the word (e.g., Durgin, 2000), has also been documented. Moreover, this effect has been observed in the case of food names and colors. For example, Nijboer et al. (2006) conducted a study in which they assessed color priming in a lexical decision task that used a color patch or word (i.e., a red patch or red word) in order to prime color-related ('tomato' and 'grass') and color-unrelated words ('timato' and 'griss'), as well as congruent (i.e., red) versus incongruent colors (i.e., blue). In Nijboer et al.'s (2006) study, color facilitated participants' decisions regarding those words that were semantically related to the color, as compared to incongruent words. In other words, color can influence decision processes and bias them toward related objects/words (see also Heurley et al., 2013). With this in mind, if, say, a flavor label and a color do not match with a consumer's previous experiences, some kind of interference can be expected when identifying and searching for a product's flavor information.

A similar color-flavor congruency effect may be expected in the context of visual search for flavor information on a product's packaging. The way in which people attend to the world around them depends on at least two attentional mechanisms: bottom-up (stimulus-driven) and top-down (goal-driven; e.g., Spence and Driver, 1994, 2004). The latter implies that, by modulating the sensitivity of the brain mechanisms that represent sensory information (top-down processing), it is possible to more efficiently explore and select one kind of stimulus over another (Wolfe and Horowitz, 2004; Knudsen, 2007). People's goals (e.g., as when searching for a flavor label) can act as a filter that facilitates selective attention to a subset of the visual information (i.e., color) competing for an observer's attention in the world (e.g., in the supermarket). A particular colorrelated word or color can activate related representations that may then exert a top-down influence that favors semantically related information. Indeed, knowing (or expecting) the color of a target can reduce search latencies (Theeuwes, 2010; Eckstein, 2011).

The present study builds on Velasco et al.'s (2014) crosscultural study of color/flavor label associations in crisps packaging. In particular, the flavor words tomato, cucumber, lemon, chicken, and BBQ were used to create both congruent and incongruent stimulus combinations. These flavors were selected because they represented the most frequent color associations across countries, although some variation from one country to another was observed in the case of lemon, chicken, and BBQ. Table 1 presents a summary of the results of one of the tasks used by Velasco et al. (2014) in which the participants had to select the color that they thought best matched the flavor label. These flavor words were first used in a visual search task (just one set size) and a variant of the go/no-go task. The aim here was to assess whether any Stroop-like effect between packaging colors and flavors was robust. While it may be reasonable to expect such an effect in pairings such as tomato and red, it is, however, less clear whether this would also be the case for previously established associations such as between chicken flavor and the color orange, or between BBQ flavor and a burgundy color (see Table 1). Moreover, it is not clear how these associations would influence search efficiency. That said, Experiment 2 was conducted and included a set-size manipulation in order to assess the influence of congruence and association strength (weaker vs. stronger) on search efficiency.

\section{Experiment 1}

\section{Methods and Materials Participants}

Thirty-two participants (17 female) aged 18-25 years $(M=20.25$ years, $\mathrm{SD}=1.88)$ from mainland China took part in this experiment. In the two experiments reported here, all of the participants were Chinese and reported normal or corrected-to-normal vision and no color blindness. They were all recruited from the subject pool of the Applied Cognitive 
TABLE 1 | Most selected colors in one of the tasks used by Velasco et al. (2014), in which the participants had to select the color that they thought best matched each flavor label.

\begin{tabular}{lll}
\hline Country & Flavor label & Color \\
\hline Colombia & BBQ & Burgundy (86.2\%) \\
Chicken & Orange (77.6\%) \\
Tomato & Red (96.6\%) \\
Cucumber & Green (91.4\%) \\
Lemon & Green (100.0\%) \\
BBina & Chicken & Burgundy (36.2\%) \\
& Tomato & Orange (44.8\%) \\
& Cucumber & Red (75.9\%) \\
& Lemon & Green (82.8\%) \\
BBQ & Yellow (65.5\%) \\
Chicken & Burgundy (87.9\%) \\
& Tomato & Orange (69.0\%) \\
& Cucumber & Red (93.1\%) \\
& Lemon & Green (96.6\%) \\
\hline
\end{tabular}

The percentages indicate the proportion of participants that selected the color.

Psychology Laboratory of the Psychology Department at Tsinghua University, Beijing, China. Each participant only took part in one of the experiments, and was either paid (20/25 Chinese Yuan in Experiments 1 and 2, respectively, depending on the duration of the experiment), or else given partial credit to fulfill the requirements of an introductory psychology course in return for taking part in the study. The experiments reported here were all approved by the Central University Research Ethics Committee of the University of Oxford, and were conducted in accordance with the ethical guidelines laid down by the Department of Experimental Psychology at the University of Oxford.

\section{Apparatus and Materials}

E-Prime 2.0 software (Psychology Software Tools, Inc.) was used to present the stimuli and to collect the data. The participants were seated in front of a 17 inch monitor (at a distance of approximately $50 \mathrm{~cm}$ from the screen). The screen had a resolution of $1024 \times 768$ pixels, and a screen refresh rate of $60 \mathrm{~Hz}$.

An image of the packaging of a fictitious brand of crisps ("Crispies") was modified using Adobe Photoshop CS4 in order to create a set of congruent and incongruent (color/flavor) packaging designs, and additional packaging designs for use in the target-absent trials (see Figure 1 for an example of the packaging stimuli used in the present study). The packages had a mean luminance of 103.73 Lumas $(S D= \pm 39)$, and were all presented against a full-screen white background.

The colors that were used were selected from RGB color codes in Adobe Photoshop CS4 as follows: burgundy 8a1d36 (RGB: 138, 29, 54), green 6fae54 (RGB: 111, 174, 84), orange e67f48 (RGB: 230, 127, 72), red d44141 (RGB: 212, 65, 65), and yellow fdd 808 (RGB: 253, 216, 8).

The experiment comprised two tasks, a visual search task and a variant of the go/no-go task. The order in which the two tasks

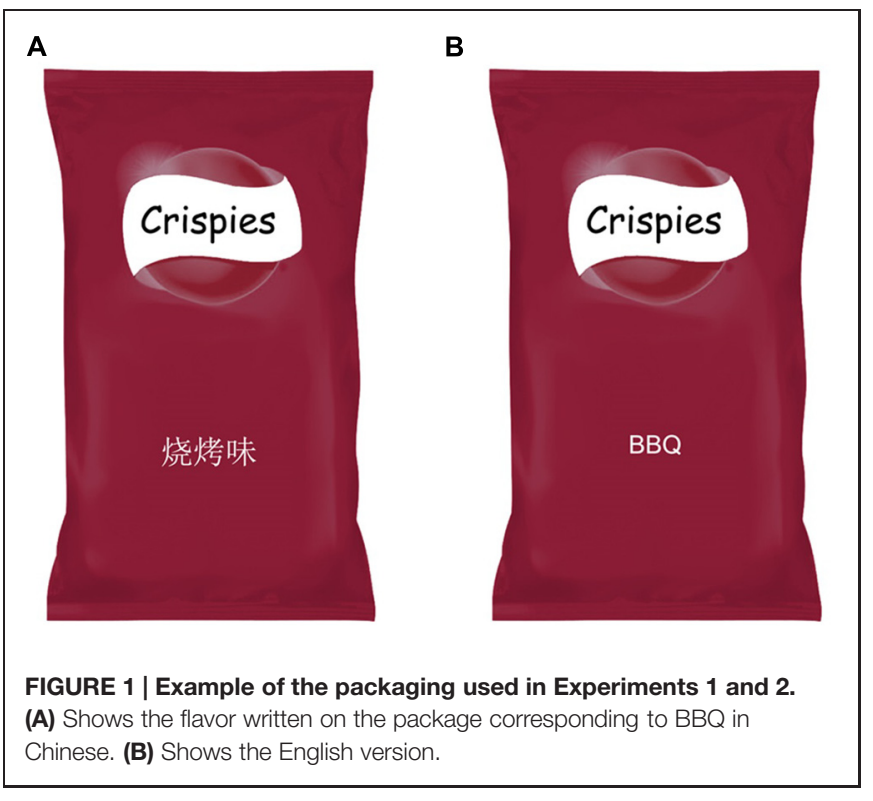

were presented was counterbalanced across participants. The targets in both tasks included congruent and incongruent stimuli. The congruent stimulus combinations included a total of five packages: BBQ flavor in burgundy, chicken flavor in orange, cucumber flavor in green, lemon flavor in yellow, and tomato flavor in red (based on the results of Velasco et al., 2014). The incongruent stimulus combinations included packages with the same flavor labels but now presented in one of the other four (incongruent) colors (i.e., BBQ crisps presented in an orange, green, yellow, or red color). The participants were not told about the congruent or incongruent associations prior to taking part in the study. In addition to the targets, a pool of 20 stimuli was created and used for the no-go and target-absent trials in the go/no-go and the visual search tasks, respectively. This pool comprised four task-irrelevant flavor labels, namely, meat, natural, original, and spice, each of which was presented against the five colors of the targets, namely, burgundy, orange, green, yellow, and tomato.

\section{Design and Procedure Visual search task}

The participants were presented with written instructions on the computer monitor saying that in every trial, they would first read a target flavor word, followed by a display containing four products, and that their task was to press one key when the target was present and another key when the target was absent. Half of the participants responded by pressing the ' $z$ ' key for target-present trials, while the other half responded by pressing the ' $m$ ' key instead (and vice versa for target-absent trials; see Figure 2A). All of the participants started the experiment with 15 practice trials, including five congruent, five incongruent, and five target-absent trials, all presented in a random order.

Four packaging designs were presented in every trial in a $2 \times 2$ array. Three were randomly assigned as distractors and the fourth 


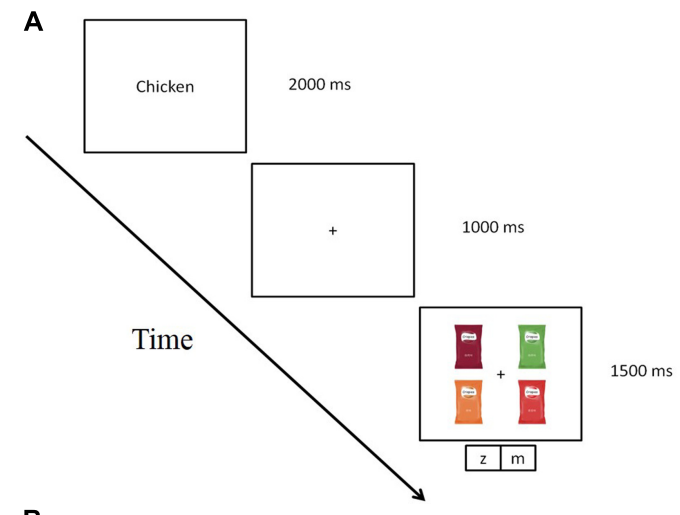

B

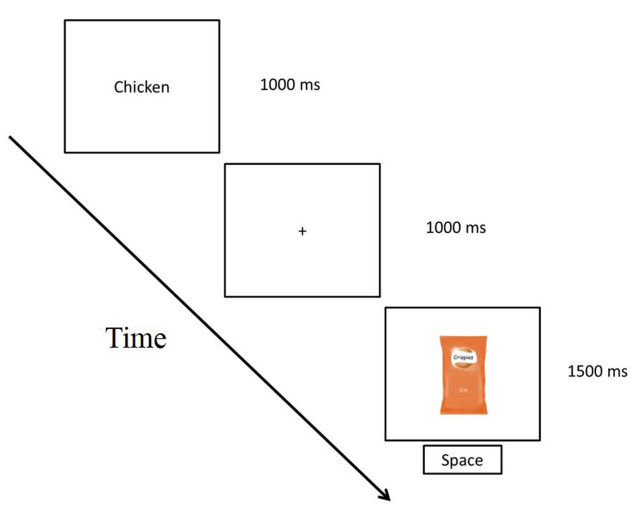

FIGURE 2 | Schematic representation of a trial in the visual search task (A) and a trial in the go/no-go task (B) in Experiment 1.

product was the target (congruent or incongruent in terms of color), or else a non-target product (in target-absent trials). Five packages, selected randomly from the pool of distractors, were used as a list from which one was picked randomly on each targetabsent trial. The incongruent packages were randomly selected from a list in which the target flavor was presented in incongruently colored packaging. The positions of the packages were randomized on every trial. Each packaging image was designed to fit within an area of $8.4^{\circ} \times 12.3^{\circ}$ of visual angle, and the written flavor names subtended approximately $0.6^{\circ} \times 0.8^{\circ}$ of visual angle. The horizontal and vertical distances between the packages consisted of 7.8 and $5.5^{\circ}$ of visual angle, respectively. After the practice trials had been completed, the main experiment started. In the main experiment, 75 congruent, 75 incongruent, and 75 target-absent trials were presented randomly, giving rise to a total of 225 trials.

\section{Go/No-Go task}

At the beginning of the task, written instructions were provided on the computer screen, followed by the practice trials. In each trial, the participants were shown a verbal flavor label (e.g., "tomato," “chicken," “cucumber," "BBQ," or "lemon," for $1000 \mathrm{~ms}$ ), followed by a central fixation cross (for $1000 \mathrm{~ms}$ ), and then the first packaging image (for a maximum of $1500 \mathrm{~ms}$ ). Each packaging image was designed to fit within an area of $15.2^{\circ}$ (height) $\times 24.5^{\circ}$ (width) of visual angle and the written flavor names subtended approximately $1.5^{\circ} \times 4.3^{\circ}$ of visual angle.

The participants' task (see Figure 2B) consisted of pressing the space bar as quickly as possible when the target was presented (either a congruent or incongruent 'go' trial) and to withhold any response if a package with a flavor label different from that of the target was presented ('no-go' trials). The experiment started with practice trials, including two congruent, two incongruent, and two target-absent trials. In these trials, accuracy feedback was given for $500 \mathrm{~ms}$ during the practice trials (the word "correct" following a correct response, "incorrect" following an incorrect response, and "no response was detected" for those trials in which the participants did not make any response). For each incongruent trial, one of the four incongruent packages designed for the targeted flavor was selected at random. No-go trials were also randomly selected from the aforementioned pool of 20 possible target-absent stimuli. After the practice blocks had been finished, the main experiment began. The main experiment comprised two blocks of six congruent, six incongruent, and six target-absent trials for each of the five flavor labels (BBQ, chicken, cucumber, lemon, and tomato), giving rise to a total of 90 trials per block and 180 for the whole experiment.

\section{Results}

\section{Visual Search Task}

The data from two of the participants were excluded from the analysis due to poor performance $(<60 \% \text { correct })^{1}$. A paired-samples $t$-test on the error rate revealed that participants made less errors in the congruent $(M=12.8 \% \mathrm{~ms}, \mathrm{SE}=1.23 \%)$ than in the incongruent $(M=22.8 \% \mathrm{~ms}, \mathrm{SE}=3.0 \%)$ condition, $t(29)=3.890, p=0.001$, Cohen's $d=0.710,95 \%$ $\mathrm{CI}=-15.2 \% \geq \mu 1-\mu 2 \geq-4.7 \%$. From the total of 4500 congruent and incongruent trials including the data from all of the participants, $802(17.8 \%)$ incorrect responses were excluded from the data analysis. In addition, those reaction time (RTs) that fell 2 SDs above or below the mean were excluded from the analysis (resulting in the removal of $2.21 \%$ of the remaining trials, meaning that a total of 3616 trials were used in the analyses).

Mean RTs were computed for correct, target-present trials across conditions (congruent and incongruent). A paired-samples $t$-test was used to assess any difference between the congruent $(M=908 \mathrm{~ms}, \mathrm{SE}=15)$ and incongruent $(M=939 \mathrm{~ms}, \mathrm{SE}=15)$ trials. This revealed a significant difference of $31 \mathrm{~ms}, t(29)=3.554, p=0.001$, Cohen's $d=0.648$, $95 \% \mathrm{CI}=-49 \geq \mu 1-\mu 2 \geq-13$ (see Figure 3A for mean RTs). As for the target-absent trials, participants made on average $18.84 \%$ ( $\mathrm{SE}=2.3 \%$ ) errors, however, no significant difference was found on the error rates between flavor labels in these trials, $F(4,116)=0.801, p=0.527, \eta_{\mathrm{p}}^{2}=0.027$. On average, the participants responded more slowly to the target-absent trials than the target-present trials $(M=1172 \mathrm{~ms}, \mathrm{SE}=4 \mathrm{~ms})$. No significant difference was found in the target-absent RTs

\footnotetext{
${ }^{1}$ The data analyses reported in Experiments 1 and 2 were also performed with these data included. Note that the pattern of results was statistically similar to the results reported in the main text.
} 

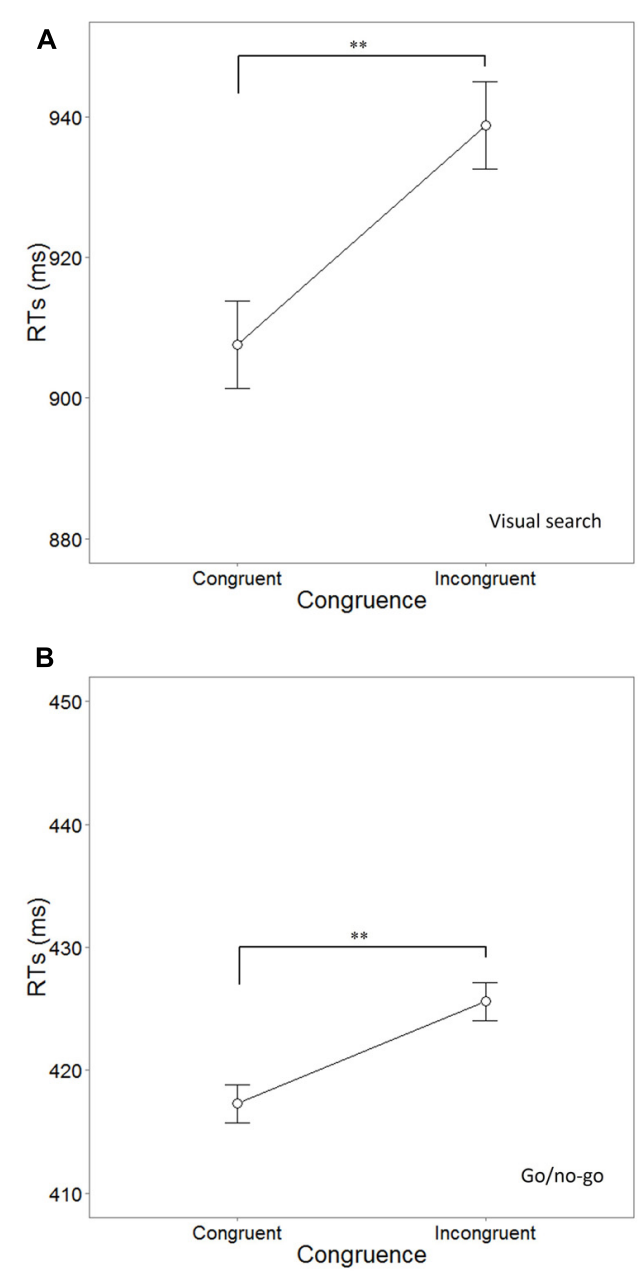

FIGURE 3 | Mean reaction times (RTs $\mathrm{ms}$ ) in each congruence condition in the visual search (A) and go/no-go task (B) tasks in

Experiment 1. The asterisks ${ }^{* *} p \leq 0.001$ ) highlight the significance of the difference and the error bars represent the SE of the means.

as a function of flavor cue, $F(4,116)=2.179, p=0.076$, $\eta_{\mathrm{p}}^{2}=0.070$

\section{Go/No-Go Task}

The data from three participants were excluded from the analysis due to their poor performance on the task ( $<60 \%$ correct). From the total of 3480 congruent and incongruent trials including the data from all remaining participants, 76 (2.1\%) incorrect responses were excluded from the analysis. A paired-samples $t$-test did not reveal a significant difference between the incongruent and congruent error rates, $t(28)=1.077, p=0.29$, Cohen's $d=0.237$. In addition, those RTs that fell 2 SDs above or below the mean were also excluded from the analysis $(4.1 \%$ of the 3404 remaining trials, for a total of 3263 used in the analyses). Mean RTs were computed for correct, target-present trials across conditions (congruent and incongruent). The difference in RTs between the congruent $(M=417 \mathrm{~ms}, \mathrm{SE}=10)$ and incongruent $(M=426 \mathrm{~ms}, \mathrm{SE}=11)$ trials was significant according to the results of a paired-samples $t$-test, $t(28)=3.845$, $p=0.001$, Cohen's $d=0.782,95 \% \mathrm{CI}=-13 \geq \mu 1-\mu 2 \geq-4$ (see Figure 3B for mean RTs). On average, participants made $6.8 \%(\mathrm{SE}=1.3 \%)$ errors in the no-go trials. A repeated measures analysis of variance (ANOVA, Greenhouse-Geisse corrected) did not reveal any difference between the error rates of the no-go trials as a function of flavor cue ("tomato," "chicken," "cucumber," "BBQ," "lemon"), $F(3.032,84.905)=0.333, p=0.855$, $\eta_{\mathrm{p}}^{2}=0.012$

\section{Discussion}

The results of both tasks provide consistent initial evidence for the idea that color/flavor label congruence facilitates visual search and that, as expected, there is a Stroop-like effect. Participant RTs were slower when responding to flavor words against an incongruent than congruent packaging colors. However, one potential limitation with the interpretation of the results of Experiment 1 relates to the fact that the congruent stimuli always consisted of the same five color/flavor combinations, while the incongruent stimuli were randomly selected from a pool of various incongruent stimuli. One may therefore wonder whether the obtained congruency effects would at least partially be attributable to the differing variety of targets in the congruent versus incongruent trials, and thus the frequency of the same congruent targets (cf. Wolfe et al., 2007; Godwin et al., 2014). Perhaps, less variety in the congruent trials might, in turn, have led to higher familiarity of the congruent stimuli and therefore faster RTs. In order to rule out this alternative explanation, Experiment 2 was conducted. Here, the variety of congruent and incongruent targets was kept the same.

In addition, Experiment 2 moved toward a more complex visual search scenario, and directly addressed two questions: (1) Does color/flavor congruence influence search efficiency? (2) Does the strength of the association between a flavor word and a color affect participants' search times to flavor words as a function of congruency? To this end, the strength of the association between a flavor word and a color, and the number of packages on the screen (set size), were manipulated. The strength of the association (or association strength) included two levels, namely, weaker and stronger associations. The two-level factor "association strength" was included based on Velasco et al.'s (2014) results. One of the levels (stronger) corresponded to those flavor labels (tomato and cucumber) to which Velasco et al.'s (2014) participants consistently selected the same color across three countries (Colombia, China, and UK) and which were highly associated with a color in China, and the other (weak) to those flavor labels (BBQ and Chicken) to which participants' associations varied as a function of country and which were only associated by some of the participants to a specific color in China (see Table 1). Presumably, some flavors have a stronger color identity due to their frequent co-occurrence with a color in the environment (e.g., cucumbers are green). Here, it was hypothesized that not only should an effect of congruency be expected, but also an effect of association strength (e.g., Velasco et al., 2014). In MacLeod 's (1991, p. 173) words: “... as the word's semantic 
association to the concept of color increases, so does its potential to interfere."

\section{Experiment 2}

\section{Methods and Materials Participants}

20 participants (10 female) aged $18-24$ years $(M=21.25$ years, $\mathrm{SD}=1.74$ ) took part in this experiment.

\section{Apparatus and Materials}

The experimental setting of Experiment 2 followed that of Experiment 1. In Experiment 2, however, exactly four pairs of congruent stimuli and four pairs of incongruent stimuli were used. In particular, the congruent combinations included chicken in orange, $\mathrm{BBQ}$ in burgundy, tomato in red, and cucumber in green. The incongruent combinations included chicken in burgundy, BBQ in green, tomato in orange, and cucumber in red. Thus, an equal number of color-flavor label pairs were used in the congruent and incongruent conditions in order to control for the differing variety of these pairings in Experiment 1. All packaging colors were set to equal luminance (122 Lumas). The colors' RGB codes in Adobe Photoshop CS4 were as follows: burgundy 732532 (RGB: 115, 37, 50), green 2d4511 (RGB: 45, 69, 17), orange 8b2800 (RGB: 139, 40, 0), and red 9e1616 $(\mathrm{RGB}=158,22,22)$. The distractors were the same as those used in Experiment 1. In addition, the experimental procedure here manipulated the set size of the packaging display, by including three, six, and nine packages in each search array.

\section{Design and Procedure}

As in Experiment 1, the participants first completed the practice trials (24 trials). The timing of each trial is illustrated in Figure 4. The participants were first presented with a fixation cross for $500 \mathrm{~ms}$, then a verbal flavor label (e.g., "chicken") for $500 \mathrm{~ms}$, then the fixation cross for $500 \mathrm{~ms}$, and finally the search display with the different packages $(3,6$, or 9) for a maximum allowable RT (after which the trial was terminated) of $3000 \mathrm{~ms}$ (due to the inclusion of larger set sizes).

After the practice trials, the main experiment started. The design consisted of two blocks of trials. Each block contained 20 congruent, 20 incongruent, and 40 target-absent trials for each set size, giving rise to 240 trials per block, and 480 trials for the whole experiment. Note that $50 \%$ of the trials were target-absent trials. When the target was absent, it was replaced by a package that was randomly selected from a list of four packages that were all labeled with "salt and vinegar" but were either orange, burgundy, green, or red. Half of the participants responded by pressing the ' $\mathrm{z}$ ' key for target-present trials, while the other half responded by pressing the ' $m$ ' key instead. The participants were given a break between the two blocks of trials. In Experiment 2, each packaging image fit within an area of $6.8 \times 11.8^{\circ}$ of visual angle. The horizontal and vertical distance between the packages was 7.8 and $1.6^{\circ}$ of visual angle for the set size with nine packages. The distances

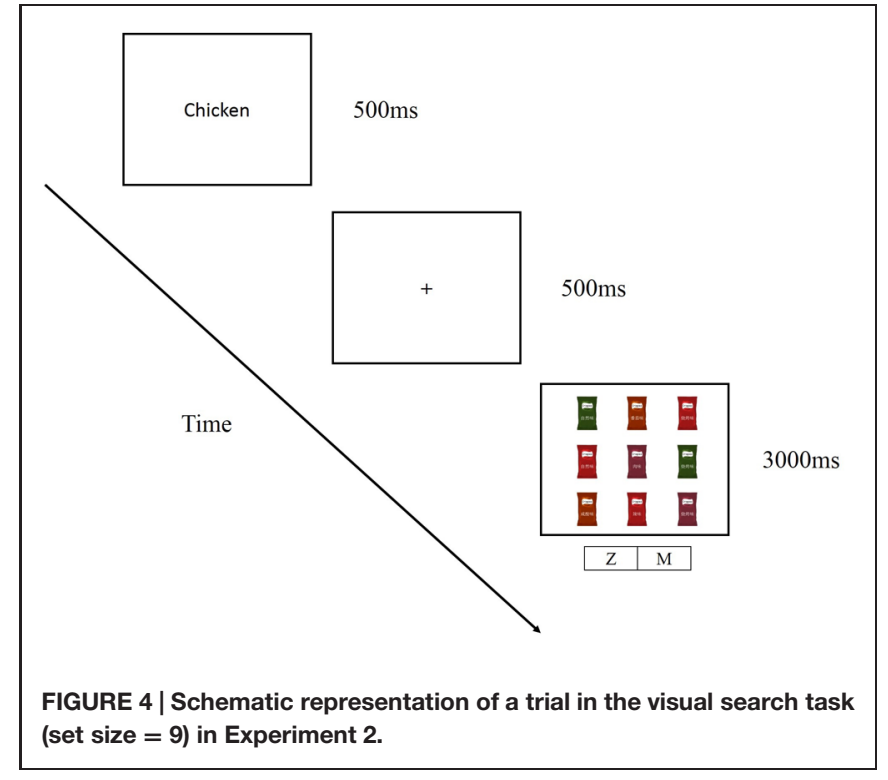

between the packages in the other set sizes (three and six) varied as a function of the position of both the target and the distractors, which were randomly assigned to any of the nine locations used in the bigger set size. The flavor names written on each package subtended approximately $1.16^{\circ} \times 2.98^{\circ}$ of visual angle.

\section{Analyses}

We performed two separate 3 (set size: three, six, nine items) $\times 2$ (association strength: weaker, stronger) $\times 2$ (congruence: congruent, incongruent) repeated-measures ANOVAs on both the error rates and RTs, and a 2 (association strength) $\times 2$ (congruence) ANOVA on the slopes that resulted from each condition. In addition, the error rates and RTs of the target-absent trials were analyzed by means of a 3 (set size: three, six, nine) $\times 2$ (association strength: weaker and stronger) ANOVA.

\section{Results \\ Error Rates}

A summary of the error rate data is presented in Table 2. Significant main effects of set size, $F(2,38)=6.326, p=0.004$, $\eta_{\mathrm{p}}^{2}=0.250$, association strength, $F(1,19)=6.774, p=0.017$, $\eta_{\mathrm{p}}^{2}=0.263$, and congruence, $F(1,19)=8.552, p=0.009$, $\eta_{\mathrm{p}}^{2}=0.210$, were found. Furthermore, the analyses revealed a significant 2-way interaction between association strength and congruence, $F(2,38)=13.327, p=0.002, \eta_{\mathrm{p}}^{2}=0.412$. Bonferronicorrected pairwise comparisons revealed that the participants made more errors in the larger set size $(M=13.13 \%, \mathrm{SE}=1.45 \%)$ than in the $3-(M=8.19 \%, \mathrm{SE}=1.06 \%)$ and 6-packaging $(M=10.31 \%, \mathrm{SE}=1.30 \%)$ set sizes $(p=0.019$ and $p=0.034$, respectively). In addition, the participants made more errors when the target was a flavor label with a stronger color association $(M=11.75 \%, \mathrm{SE}=1.11 \%)$ than when it was one of those with a weaker association $(M=9.33 \%, \mathrm{SE}=1.10 \%$, $p=0.017)$. Similarly, the participants made more mistakes in the incongruent $(M=12.17 \%, \mathrm{SE}=1.06)$ than in the congruent $(M=8.92 \%, \mathrm{SE}=1.23 \%)$ condition $(p=0.009)$. As for 
TABLE 2 | Error rates for both target-present, congruent, and incongruent, trials, as well as target-absent trials, as a function of both association strength and set size.

\begin{tabular}{lllll}
\hline Congruence & \multirow{2}{*}{$\begin{array}{l}\text { Association } \\
\text { strength }\end{array}$} & \multicolumn{3}{c}{ Set size (ER\%) } \\
\cline { 3 - 5 } & & $\mathbf{3}$ & $\mathbf{6}$ & $\mathbf{9}$ \\
\hline Congruent & Weaker & 6.75 & 7.00 & 13.00 \\
& Stronger & 6.00 & 9.75 & 11.00 \\
Incongruent & Weaker & 8.00 & 9.50 & 11.75 \\
& Stronger & 12.00 & 15.00 & 16.75 \\
Target-absent & Weaker & 6.38 & 7.88 & 11.00 \\
& Stronger & 6.75 & 9.25 & 10.63 \\
& & & &
\end{tabular}

the interaction term, it was found that, in the incongruent conditions, the participants made more errors when responding to the flavor labels with a stronger color association $(M=14.58 \%$, $\mathrm{SE}=1.49 \%)$, than those with a weaker association $(M=9.75 \%$, $\mathrm{SE}=1.31 \% ; p=0.004)$.

The analysis of the error data (using the Greenhouse-Geisser correction) of the target-absent trials only revealed a significant main effect of set size, $F(1.529,29.045)=6.605, p=0.008$, $\eta_{\mathrm{p}}^{2}=0.258$ (see Table 2). The participants made fewer errors in the smallest packaging set size $(M=6.6 \%, \mathrm{SE}=2.4 \%)$ than in the 9-packaging set size $(M=10.8 \%, \mathrm{SE}=3.1 \%$; $p=0.012)$. A marginally significant difference between the 3 - and the 6packaging $(M=8.6 \%, \mathrm{SE}=2.9 \%)$ set sizes was also observed $(p=0.058)$.

\section{Reaction Times}

From the 4800 congruent and incongruent trials from all of the participants, 506 trials with incorrect responses (10.54\%) were excluded from the RT analysis. In addition, those RTs falling 2 SDs either above or below the mean of each set size condition's RTs were excluded from the data analysis (3.98\% of the remaining trials, leaving 4123 trials in the final analyses). The results revealed a significant main effect of set size, $F(2,38)=338.946$, $p<0.001, \eta_{\mathrm{p}}^{2}=0.947$, and congruence, $F(1,19)=37.073$, $p<0.001, \eta_{\mathrm{p}}^{2}=0.661$, and a significant two-way interaction between association strength and congruence, $F(1,19)=5.978$, $p=0.024, \eta_{\mathrm{p}}^{2}=0.239$. In addition, the results also revealed a marginally significant interaction between set size and congruence, $F(2,38)=3.195, p=0.052, \eta_{\mathrm{p}}^{2}=0.144$. No significant effects were found for association strength, the interaction between set size and association strength, or the interaction between set size, association strength, and congruence (see Figure 5 for a visualization of the results).

Bonferroni-corrected pairwise comparisons revealed that the participants responded faster to the targets in the set size with three packages $(M=910 \mathrm{~ms}, \mathrm{SE}=21)$ than to those in the other two set sizes, and more rapidly to the targets in the set size with six packages $(M=1179 \mathrm{~ms}, \mathrm{SE}=30)$ than to those in the set size with nine packages $(M=1339 \mathrm{~ms}, \mathrm{SE}=30, p<0.001$, for all comparisons). Moreover, it was found that the participants responded more rapidly to the congruent $(M=1112 \mathrm{~ms}, \mathrm{SE}=24)$ than the incongruent trials $(M=1174 \mathrm{~ms}, \mathrm{SE}=28$; $p<0.001)$. As for

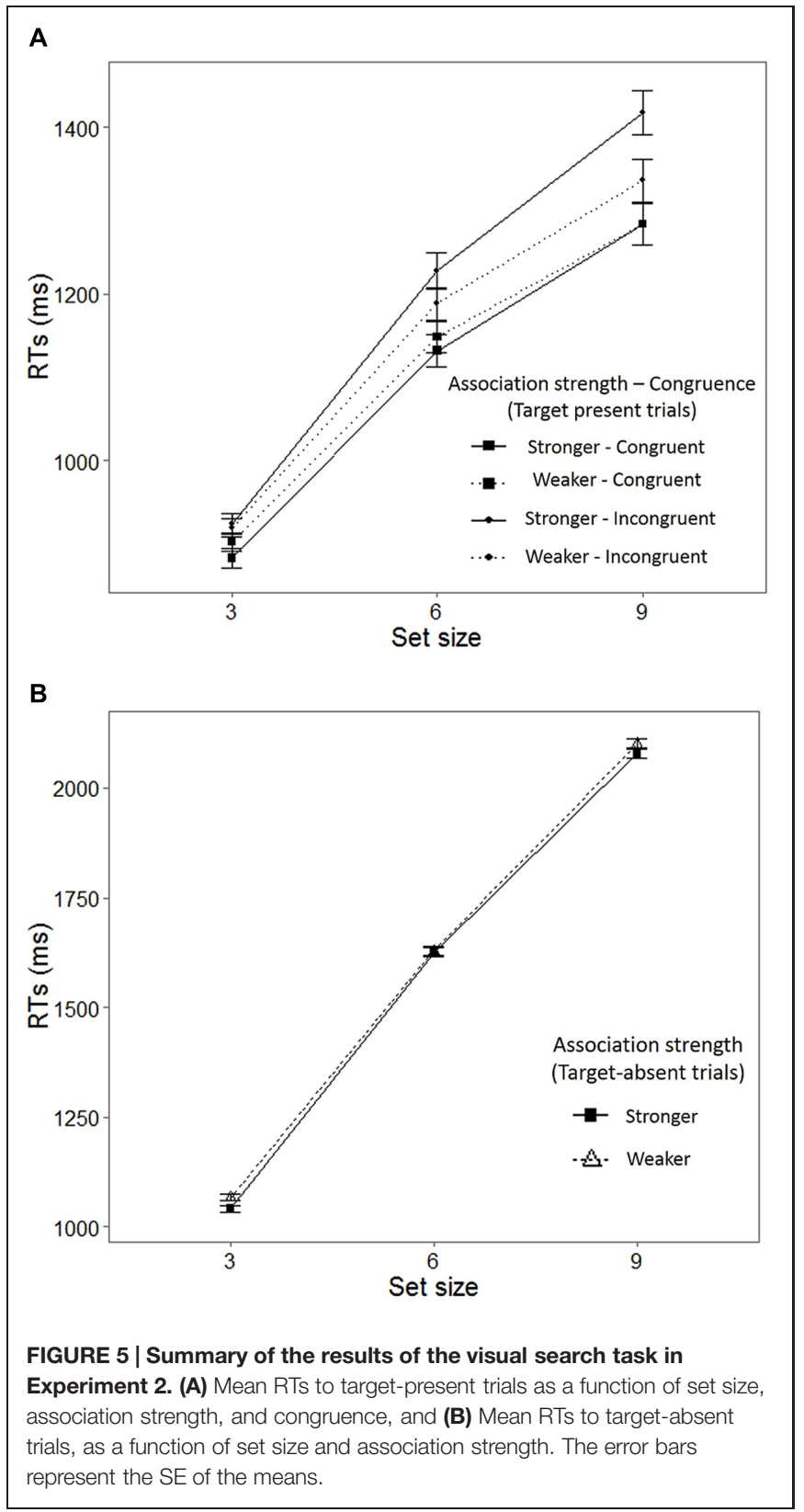

the interaction between association strength and congruence, the analyses revealed that, in the incongruent conditions, the participants responded more rapidly to the flavor labels with a weaker color association $(M=1149 \mathrm{~ms}, \mathrm{SE}=29)$ than to those with a stronger color association $(M=1191 \mathrm{~ms}, \mathrm{SE}=29 ; p=0.028)$.

The analysis of the RT data of the target-absent trials (see Figure 5B) only revealed a significant effect of set size (note that the Greenhouse-Geisser correction is used), $F(1.226$, $23.301)=725.604, p<0.001, \eta_{p}^{2}=0.974$. The participants identified that a target was absent faster in the 3-packaging set size, followed by the 6-, and 9-packaging set sizes $(p<0.001$, for all comparisons).

Details of the mean RTs, slopes, and intercepts for each condition are shown in Table 3 (see also Figure 5). A two 
TABLE 3 | Mean reaction times (RTs; in ms) as a function of set size, association strength, and congruence in Experiment 2.

\begin{tabular}{lllllll}
\hline \multirow{2}{*}{ Congruence } & \multirow{2}{*}{$\begin{array}{l}\text { Association } \\
\text { Strength }\end{array}$} & \multicolumn{3}{c}{ Set size (mean RTs) } & Slope & Intercept \\
\cline { 3 - 5 } & & $\mathbf{3}$ & $\mathbf{6}$ & $\mathbf{9}$ & & \\
\hline \multirow{2}{*}{ Congruent } & Weaker & 919 & 1148 & 1284 & 64 & 729 \\
& Stronger & 923 & 1132 & 1284 & 67 & 697 \\
\multirow{2}{*}{ Incongruent } & Weaker & 901 & 1188 & 1333 & 70 & 730 \\
& Stronger & 883 & 1228 & 1418 & 83 & 694 \\
\multirow{2}{*}{ Target-absent } & Weaker & 1067 & 1629 & 2099 & 172 & 566 \\
& Stronger & 1041 & 1625 & 2078 & 172 & 544 \\
\hline
\end{tabular}

The last two columns provide the mean slopes and intercepts (all significant, $p<0.001)$.

(association strength: weaker and stronger) $\times 2$ (congruence: congruent and incongruent) ANOVA was performed on the slope values. The analysis revealed significant main effects of association strength, $F(1,19)=5.925, p=0.025, \eta_{\mathrm{p}}^{2}=0.238$, and congruence, $F(1,19)=5.052, p=0.037, \eta_{\mathrm{p}}^{2}=0.210$. The interaction was not significant. The slopes were steeper when the participants responded to the flavor words with a stronger $(M=76, \mathrm{SE}=3)$ than a weaker $(M=67, \mathrm{SE}=4)$ association strength $(p=0.025)$. Likewise, the slopes were steeper in the incongruent $(M=77, \mathrm{SE}=4)$ than the congruent $(M=66$, $\mathrm{SE}=3)$ combination $(p=0.037)$.

\section{Discussion}

The results of Experiment 2 therefore replicate those of Experiment 1 while providing further evidence to suggest that the effect is still present across different set sizes. In addition, they support the idea that when the packaging color is incongruent those flavor words to which a color is more strongly associated will be harder to find than those which are less strongly associated to a color. Importantly, search efficiency is affected by both congruence and association strength, with congruent combinations producing more efficient searches on the one hand, and flavor words with a weaker association strength on the other. There are several points to be mentioned here: First, color is a preattentively processed feature that can guide attention more efficiently toward a target, producing near-flat search slopes (Wolfe and Horowitz, 2004). In the context of the present study though, when the color of the packaging is congruent but the word is not the target (mismatch), participants then have to move to another target, thus, producing slower, more effortful, searches (see also Grossberg et al., 1994). Moreover, the strength of the association may also be key as participant's previous associations between a flavor and a word may then determine which color would guide attention. Finally, while the aforementioned points may help to explain why color does not seem to produce efficient searches, it is also worth mentioning that the packages used as distractors could also have had a color that was semantically congruent with the target (though with a distractor flavor label). Such an element may have increased noise (cf. Wolfe, 2005), and thus made search less efficient as if, say, each search trial had had categorically different colors (target-distractor similarity).

\section{General Discussion}

The two experiments reported in the present study provide evidence that the visual search for product packaging is influenced by color-flavor congruence and association strength. Specifically, participants searched for the target flavor labels more rapidly when they were presented in a packaging having a color that was congruent (vs. incongruent) with the cued flavor. These results are consistent with the idea that color can facilitate participants' responses toward concepts that are semantically related to colors in a Stroop-like fashion (e.g., MacLeod, 1991; Durgin, 2000; Nijboer et al., 2006; Richter and Zwaan, 2009). It is possible that a particular word can activate object-related representations that may then exert some kind of top-down influence on the tasks. That is, when the actual object fits the participant's expectations, a facilitation effect can be observed. In addition, incongruent colors tend to interfere more with flavor words that have a stronger color identity (e.g., cucumbers tend to be green) than with those with a weaker color identity (e.g., chicken can be orange in the context of crisps).

The results of the two experiments reported here are also consistent with the literature on the Stroop and the reverse Stroop effects (see MacLeod, 1991, for a review). According to this literature, a color word can influence the way in which people respond to the font color and a physical color can also influence participants' responses to a color word (e.g., Richter and Zwaan, 2009). A particular word or color can activate related representations that may then exert a top-down influence on the tasks. In other words, the visual elements that people link to a particular food or food word can influence the visual search for food products as a function of congruency.

The interaction between association strength and congruence that was observed in Experiment 2 is comparable to one of the experiments (Experiment 3) reported by Berthet et al. (2011). By using both an affective priming and a Stroop task, they found an interaction between relevant information strength (e.g., how strongly a target is associated with a color) and congruence (valence-picture for the affective priming task and color-object for the Stroop task). In particular, Berthet et al. (2011) documented a significant difference between congruent and incongruent trials in the stronger condition, but not in the weaker one. While in the present study a difference between congruent and incongruent trials was found both in the weaker and stronger condition, it appears as if the difference is larger in the stronger condition than in the weaker one. This, in turn, suggests greater interference, which is reflected in the difference between weaker and stronger conditions in the incongruent trials.

Here it is important to mention that the pattern of performance observed in the target-absent trials in Experiment 2 should be interpreted with some degree of caution. When the target was absent, one of four possible packages, labeled with "salt and vinegar" and either orange, burgundy, green, or red color, was randomly selected and used in the trial. As only one flavor label was used, it is possible that our participants may have adopted a strategy whereby they responded 'absent' as soon as they saw 'salt and vinegar' (and hence a target), with the second target being the intended target for that trial. However, one finding that 
suggests that this strategy was not adopted by the participants is the observation that the mean slope of the target-absent trials was more than twice as steep as the slopes of both the congruent and incongruent target present trials. This slope pattern is typically observed for target-absent versus target-present trials (cf. Wolfe, 1998). In addition, the results regarding the main hypothesis of this study would be unaffected by such a response strategy; and the main hypothesis is therefore still supported by the results of both experiments.

The results reported here therefore highlight the importance of flavor labeling and color congruence when it comes to classifying and searching for flavor information. As suggested by Velasco et al. (2014), it is likely that the presented color/flavor label associations are learnt by internalizing the statistical regularities in the environment (i.e., common pairings between colors and flavors). Such regularities also extend to the market place (e.g., Labrecque and Milne, 2013), and frequently, object/color co-occurrences can be internalized by the consumer and subsequently influence information processing as a function of the level of congruency (e.g., Clydesdale, 1991; Piqueras-Fiszman et al., 2012; Velasco et al., 2014). The experimental approach outlined here may be useful for those marketing practitioners interested in launching a new product, or those thinking about changing the color of their current product offerings. By assessing the effects of specific color-related words such as flavor words, marketers may be able to understand which colors may or may not interfere with such words.

The Stroop-like effect observed here is also compatible with the literature on visual search. Eimer (2014) recently put forward a model of selective attention designed to account for the existence of four key stages in visual search, which include preparation, guidance, selection, and finally identification. Preparation refers to the activation of representations, or 'target templates' in working memory, related to the target information, before the actual search; working memory is in charge of holding the object's sensory features relevant to the search task, that are not directly accessible via the senses. Guidance, on the other hand, refers to the process of gathering target-related information in parallel, while selection refers to the assignment of resources to the potential targets, and identification, to the process of binding the object's features for the subsequent recognition of the target's identity. The color associated with a flavor may therefore be represented in working memory while searching for a specific flavor label, and potentially, those flavor labels that are presented in a manner that is consistent with the statistical regularities of the environment (see Spence, 2011 and Parise

\section{References}

Berthet, V., Kop, J. L., and Kouider, S. (2011). Response interference in compatibility tasks: effects of target strength in affective priming and Stroop. Exp. Psychol. 58, 257. doi: 10.1027/1618-3169/a000092

Clydesdale, F. M. (1991). Color perception and food quality. J. Food Qual. 14, 61-74. doi: 10.1111/j.1745-4557.1991.tb00047.x

Duncan, J., and Humphreys, G. W. (1989). Visual search and stimulus similarity. Psychol. Rev. 96, 433-458. doi: 10.1037/0033-295X.96.3.433

Durgin, F. H. (2000). The reverse Stroop effect. Psychon. Bull. Rev. 7, 121-125. doi: $10.3758 /$ BF03210730 and Spence, 2013), such as cucumber in green, may be easier to classify and hence to search for (cf. Vickery et al., 2005). Importantly, search efficiency is affected by both congruence and the strength of the association. This result is particularly intriguing given the existing evidence suggesting that search is guided by preattentive features such as color, as suggested by Eimer (2014; see also Wolfe, 2007). There is, however, research suggesting that when a mismatch is found (e.g., an incongruent color after a flavor cue) a deeper search is performed, which could influence RTs (Grossberg et al., 1994). Furthermore, as the color of the distractors could also match participants' color representation of a flavor, search may have been less efficient.

It will be interesting in future research to look at how color/flavor associations affect a person's search for a particular product flavor (i.e., would red be associated with tomato in a yogurt, say?). Another interesting direction for future research concerns target/distractor similarity, since that has been shown to influence search times (e.g., Duncan and Humphreys, 1989). In effect, products are generally presented in supermarket shelves, grouped by brands and thus by their visual appearance. Since the position of the target and distractors was always randomized and the distractors were randomly selected from a pool of distractors, some trials included two or more packages with the same color; thus it is difficult to say whether target distractor similarity exerted any influence on the search process. Anyhow, the supermarket aisle can be thought of as representing a highly complex visual search environment, in which color/flavor associations may play a key role.

In summary, we provide evidence for the idea that the congruence between color and flavor is a key element when searching for flavor information. We believe that the task used here could be fruitful for both researchers and marketing practitioners. For the former, this type of task may help to assess the influence of crossmodal associations captured in language (e.g., colors and flavor words) on search efficiency. For the latter, it may provide useful when assessing which color to use when, for example, a new product is launched.

\section{Acknowledgments}

Charles Spence would like to acknowledge the Rethinking the Senses grant from the AHRC (UK; AH/L007053/1). This research was also supported by National Natural Science Foundation of China (Grant No. 71472106) awarded to Xiaoang Wan.

Eckstein, M. P. (2011). Visual search: a retrospective. J. Vis. 11, 1-36. doi: $10.1167 / 11.5 .14$

Eimer, M. (2014). The neural basis of attentional control in visual search. Trends Cogn. Sci. 18, 526-535. doi: 10.1016/j.tics.2014.05.005

Garber, L. L. Jr., Hyatt, E. M., and Starr, R. G. Jr. (2000). Placing food color experimentation into a valid consumer context. J. Food Prod. Mark. 7, 3-24. doi: 10.1300/J038v07n03_02

Garber, L. L. Jr., Hyatt, E. M., and Starr, R. G. Jr. (2001). The effects of food color on perceived flavor. J. Mark. Theory Pract. 8, 59-72.

Godwin, H. J., Menneer, T., Cave, K. R., Thaibsyah, M., and Donnelly, N. (2014). The effects of increasing target prevalence on information processing during 
visual search. Psychon. Bull. Rev. doi: 10.3758/s13423-014-0686-2 [Epub ahead of print].

Grossberg, S., Mingolla, E., and Ross, W. D. (1994). A neural theory of attentive visual search: interactions of boundary, surface, spatial, and object representations. Psychol. Rev. 101, 470. doi: 10.1037/0033-295X.101.3.470

Heckler, S. E., and Childers, T. L. (1992). The role of expectancy and relevancy in memory for verbal and visual information: what is incongruency? J. Consum. Res. 18, 475-492. doi: 10.1086/209275

Heurley, L. P., Brouillet, T., Chesnoy, G., and Brouillet, D. (2013). Color perception involves color representations firstly at a semantic level and then at a lexical level. Cogn. Process. 14, 19-29. doi: 10.1007/s10339-012-0527-z

Hutchings, J. B. (1977). The importance of visual appearance of foods to the food processor and the consumer. J. Food Qual. 1, 267-278. doi: 10.1111/j.17454557.1977.tb00945.x

Klein, G. S. (1964). Semantic power measured through the interference of words with color-naming. Am. J. Psychol. 77, 576-588. doi: 10.2307/1420768

Klopfer, D. S. (1996). Stroop interference and color-word similarity. Psychol. Sci. 7, 150-157. doi: 10.1111/j.1467-9280.1996.tb00348.x

Knudsen, E. I. (2007). Fundamental components of attention. Annu. Rev. Neurosci. 30, 57-78. doi: 10.1146/annurev.neuro.30.051606.094256

Labrecque, L. L., and Milne, G. R. (2013). To be or not to be different: exploration of norms and benefits of color differentiation in the marketplace. Mark. Lett. 24, 165-176. doi: 10.1007/s11002-012-9210-5

Lewis, D. E., Pearson, J., and Khuu, S. K. (2013). The color "fruit": object memories defined by color. PLoS ONE 8:e64960. doi: 10.1371/journal.pone.0064960

MacLeod, C. M. (1991). Half a century of research on the Stroop effect: an integrative review. Psychol. Bull. 109, 163-203. doi: 10.1037/0033-2909.109. 2.163

Madden, T. J., Hewett, K., and Roth, M. S. (2000). Managing images in different countries: a cross-national study of color meanings and preferences. J. Int Mark. 8, 90-107. doi: 10.1509/jimk.8.4.90.19795

Mandler, G. P. (1982). “The structure of value: accounting for taste," in Proceedings of the Affect and Cognition. The 17th Annual Carnegie Symposium on Cognition, eds M. S. Clark and S. T. Fiske (Hillsdale, NJ: Lawrence Erlbaum), 3-36.

Miller, E. G., and Kahn, B. E. (2005). Shades of meaning: the effect of color and flavor names on consumer choice. J. Consum. Res. 32, 86-92. doi: 10.1086/ 429602

Nijboer, T. C., van Zandvoort, M. J., and de Haan, E. H. (2006). Seeing red primes tomato: evidence for comparable priming from colour and colour name primes to semantically related word targets. Cogn. Process. 7, 269-274. doi: 10.1007/s10339-006-0153-8

Parise, C., and Spence, C. (2013). "Audiovisual cross-modal correspondences in the general population," in The Oxford Handbook of Synaesthesia, eds J. Simner and E. Hubbard (Oxford: Oxford University Press), 790-815. doi: 10.1093/oxfordhb/9780199603329.013.0039

Peracchio, L. A., and Tybout, A. M. (1996). The moderating role of prior knowledge in schema-based product evaluation. J. Consum. Res. 23, 177-192. doi: $10.1086 / 209476$

Piqueras-Fiszman, B., and Spence, C. (2011). Crossmodal correspondences in product packaging. Assessing colour-flavour correspondences for potato chips (crisps). Appetite 57, 753-757. doi: 10.1016/j.appet.2011.07.012

Piqueras-Fiszman, B., Velasco, C., and Spence, C. (2012). Exploring implicit and explicit crossmodal colour-flavour correspondences in product packaging. Food Qual. Prefer. 25, 148-155. doi: 10.1016/j.foodqual.2012.02.010

Proctor, R. W. (1978). Sources of color-word interference in the Stroop colornaming task. Percept. Psychophys. 23, 413-419. doi: 10.3758/BF03204145

Richter, T., and Zwaan, R. A. (2009). Processing of color words activates color representations. Cognition 111, 383-389. doi: 10.1016/j.cognition.2009.02.011

Scheibe, K. E., Shaver, P. R., and Carrier, S. C. (1967). Color association values and response interference on variants of the Stroop test. Acta Psychol. 26, 286-295. doi: 10.1016/0001-6918(67)90028-5

Schoormans, J. P., and Robben, H. S. (1997). The effect of new package design on product attention, categorization and evaluation. J. Econ. Psychol. 18, 271-287. doi: 10.1016/S0167-4870(97)00008-1

Shankar, M. U., Levitan, C. A., and Spence, C. (2010). Grape expectations: the role of cognitive influences in colour-flavour interactions. Conscious. Cogn. 19, 380-390. doi: 10.1016/j.concog.2009.08.008

Spence, C. (2011). Crossmodal correspondences: a tutorial review. Atten. Percept. Psychophys. 73, 971-995. doi: 10.3758/s13414-010-0073-7
Spence, C. (2012). Managing sensory expectations concerning products and brands: capitalizing on the potential of sound and shape symbolism. J. Consum. Psychol. 22, 37-54. doi: 10.1016/j.jcps.2011.09.004

Spence, C. J., and Driver, J. (1994). Covert spatial orienting in audition: exogenous and endogenous mechanisms. J. Exp. Psychol. Hum. Percept. Perform. 20, 555-574. doi: 10.1037/0096-1523.20.3.555

Spence, C., and Driver, J. (eds). (2004). Crossmodal Space and Crossmodal Attention. Oxford: Oxford University Press. doi: 10.1093/acprof:oso/9780198524861.001.0001

Spence, C., Levitan, C. A., Shankar, M. U., and Zampini, M. (2010). Does food color influence taste and flavor perception in humans? Chemosens. Percept. 3, 68-84. doi: 10.1007/s12078-010-9067-z

Spence, C., Velasco, C., and Knoeferle, K. (2014). A large sample study on the influence of the multisensory environment on the wine drinking experience. Flavour 3, 8. doi: 10.1186/2044-7248-3-8

Stevenson, R. J. (2009). The Psychology of Flavour. Oxford: Oxford University Press. doi: 10.1093/acprof:oso/9780199539352.001.0001

Stillman, J. A. (1993). Color influences flavor identification in fruit-flavored beverages. J. Food Sci. 58, 810-812. doi: 10.1111/j.1365-2621.1993.tb09364.x

Tanaka, J. W., and Presnell, L. M. (1999). Color diagnosticity in object recognition. Percept. Psychophys. 61, 1140-1153. doi: 10.3758/BF03207619

Theeuwes, J. (2010). Top-down and bottom-up control of visual selection. Acta Psychol. 135, 77-99. doi: 10.1016/j.actpsy.2010.02.006

Velasco, C., Jones, R., King, S., and Spence, C. (2013). Assessing the influence of the multisensory environment on the whisky drinking experience. Flavour 2, 23. doi: 10.1186/2044-7248-2-23

Velasco, C., Wan, X., Salgado-Montejo, A., Woods, A., Onate, G., Mi, B., et al. (2014). The context of colour-flavour associations in crisps packaging: a crosscultural study comparing Chinese, Colombian, and British consumers. Food Qual. Prefer. 38, 49-57. doi: 10.1016/j.foodqual.2014.05.011

Vickery, T. J., King, L. W., and Jiang, Y. (2005). Setting up the target template in visual search. J. Vis. 5, 81-92. doi: 10.1167/5.1.8

Wan, X., Velasco, C., Michel, C., Mu, B., Woods, A. T., and Spence, C. (2014a). Does the type of receptacle influence the crossmodal association between colour and flavour? A cross-cultural comparison. Flavour 3, 3. doi: 10.1186/2044-72483-3

Wan, X., Zhou, X., Mu, B., Du, D., Velasco, C., Michel, C., et al. (2014b). Crossmodal expectations of tea color based on flavor: a preliminary study with naïve assessors. J. Sens. Stud. 29, 285-293. doi: 10.1111/joss.12102

Wolfe, J. (2005). "Guidance of visual search by preattentive information," in Neurobiology of Attention, eds L. Itti, G. Ress, and J. K. Tsotos (San Diego, CA: Elsevier), 101-104.

Wolfe, J. M. (1998). What do 1,000,000 trials tell us about visual search? Psychol. Sci. 9, 33-39. doi: 10.1111/1467-9280.00006

Wolfe, J. M. (2007). "Guided search 4.0: current progress with a model of visual search," in Integrated Models of Cognitive Systems, ed. W. Gray (New York, NY: Oxford), 99-119.

Wolfe, J. M., and Horowitz, T. S. (2004). What attributes guide the deployment of visual attention and how they do it? Nat. Rev. Neurosci. 5, 495-501. doi: $10.1038 /$ nrn 1411

Wolfe, J. M., Horowitz, T. S., Van Wert, M. J., Kenner, N. M., Place, S. S., and Kibbi, N. (2007). Low target prevalence is a stubborn source of errors in visual search tasks. J. Exp. Psychol. Gen. 136, 623-638.

Zampini, M., Sanabria, D., Phillips, N., and Spence, C. (2007). The multisensory perception of flavor: assessing the influence of color cues on flavor discrimination responses. Food Qual. Prefer. 18, 975-984. doi: 10.1016/j.foodqual.2007.04.001

Conflict of Interest Statement: The authors declare that the research was conducted in the absence of any commercial or financial relationships that could be construed as a potential conflict of interest.

Copyright (c) 2015 Velasco, Wan, Knoeferle, Zhou, Salgado-Montejo and Spence. This is an open-access article distributed under the terms of the Creative Commons Attribution License (CC BY). The use, distribution or reproduction in other forums is permitted, provided the original author(s) or licensor are credited and that the original publication in this journal is cited, in accordance with accepted academic practice. No use, distribution or reproduction is permitted which does not comply with these terms. 